Abstract P3-S6.03 Table 1 Seroreversion TT Congenital Syphillis

\begin{tabular}{|c|c|c|c|c|}
\hline Case* & Maternal stage/GA at treatment & Neonatal/infant diagnostic features & Infant age at treatment (GA at birth) & Infant age /final serologic results \\
\hline 1 & Primary/postpartum & Abnormal CSF (4) & Birth (34 weeks) & 7 months/RPR NR, TPPA NR, FTA-ABS NR \\
\hline \multirow[t]{4}{*}{2} & Primary/postpartum & Abnormal CSF (3) & Birth (unknown) & 14 months/RPR NR, TPPA NR \\
\hline & & Abnormal long bone radiographs & & \\
\hline & & Intraventricular haemorrhage & & \\
\hline & & Fetal hydrops & & \\
\hline 3 & Primary/postpartum & Abnormal CSF (3) & Birth (38 weeks) & 10 months/syphilis EIA negative \\
\hline 4 & Early latent/postpartum & Abnormal CSF (3) & Birth ( 30 weeks) & 5 months/syphilis EIA negative \\
\hline 5 & Early latent/34 weeks GA & $\begin{array}{l}\text { Abnormal CSF ( } 3 \text { ) Abnormal long bone } \\
\text { radiographs }\end{array}$ & Birth (37 weeks) & 12 mos/syphilis EIA negative \\
\hline 6 & Primary/postpartum & Abnormal CSF (3) & Birth (38 weeks) & 18 months/syphilis EIA negative \\
\hline 7 & Early latent/postpartum & Abnormal CSF (4) & Birth (36 weeks) & 19 months/syphilis EIA negative \\
\hline 8 & Secondary/28 weeks GA & $\begin{array}{l}\text { Abnormal CSF (4) Intrauterine anaemia, } \\
\text { hydrops, cardiomegaly, ascites. Positive } \\
\text { syphilis PCR from intrauterine fetal blood }\end{array}$ & Birth (36 weeks) & 13 months/syphilis EIA negative \\
\hline
\end{tabular}

CSF, cerebrospinal fluid; GA, gestational age; NR, non reactive; R, reactive; EIA, enzyme immunoassay; RPR, rapid plasma reagin; TPPA,Treponema pallidum particle agglutination; FTA-ABS, fluorescent treponemal antibody absorbed; PCR, polymerase chain reaction.

*Number of CSF abnormalities (elevated WBC, RBC or protein, low glucose, reactive VDRL).

under 18 month serologic follow-up, one had persistently reactive TT (21 months) and four had reactive TT at the end of their followup period (ages 11, 12, 13 and 15 months). 3/5 cases with persistently reactive TT were treated with 9-10 days of intravenous penicillin within $0-2$ days of birth, 1 at 3 months of age and 1 at 8 months of age. In $4 / 5$ of these cases, the RPR had reverted to non reactive at the end of the follow-up period while in the 5th case (treated at 8 months), the RPR declined from a titre of 1:4096 dilutions at birth to 1:64 dilutions at 11 months of age. The remaining eight cases had negative TTs, as summarised in the table. All were treated with 10 days of intravenous penicillin (except case \#2 treated with 9 days) see Abstract P3-S6.03 table 1.

Conclusions As with early treatment of primary syphilis cases, seroreversion of TT can occur in cases meeting clinical and laboratory criteria for congenital syphilis. Seroreversion was observed with older TT such as TPPA and FTA-ABS as well as the newer syphilis EIA.

\section{P3-S6.04 USE OF A POINT OF CARE TEST DEVICE TO DETECT SYPHILIS IN A STD CLINIC}

doi:10.1136/sextrans-2011-050108.478

${ }^{1} \mathrm{M}$ Barnes, ${ }^{2} \mathrm{D}$ Ducan, ${ }^{3} \mathrm{P}$ Agreda, ${ }^{3} \mathrm{Y}$ H Hsieh, ${ }^{3} \mathrm{~J}$ Holden, ${ }^{3} \mathrm{~A}$ Rompalo, ${ }^{3} \mathrm{C}$ Gaydos. ${ }^{1}$ Johns Hopkins, Baltimore, USA; ${ }^{2}$ Health Department, Baltimore, USA; ${ }^{3}$ Johns Hopkins University, Baltimore, USA

Background Diagnosis of syphilis is problematic and an accurate rapid point of care (POC) test could be useful in busy STD clinics. There are no FDA Cleared POC tests for Syphilis serology in the USA.

Objective To determine the performance of a new, rapid point of care (POC) test, Syph-Check, which is not yet FDA cleared, for the serological diagnosis of Treponema pallidum in female and male STD patients.

Methods Men and women $>18$ yr visiting the Baltimore City Health Department STD clinic were consented to enrol in a trial to determine the accuracy of a new, innovative POC test for syphilis (Veda, manufactured in France) that used a cassette format to test syphilis serology. The Syph-Check One-Step Syphilis test is a point of care, rapid immunoassay screening test for qualitative detection of IgG and IgM antibodies to Treponema pallidum in finger stick blood, plasma, and serum. This product can be used as an initial screening test or as a confirmatory diagnostic test, but is not FDA cleared for use in screening blood or plasma donors. The assay was performed in the STD clinic, required only $20 \mathrm{~min}$ to perform, and required no instrumentation. RPR and TPPA tests were performed to determine the sensitivity and specificity of the Syph-Check POC test.

Results 194 men and 205 women were enrolled. Of the 399 samples tested, 33 were positive and 366 were negative by the Syph-Check. There were 14 positives and 385 negatives by RPR confirmatory testing. Overall sensitivity compared to RPR testing was $85.7 \%$ (95\% CI $60.3 \%$ to $97.5 \%$ ) and specificity was $94.5 \%$ (95\% CI $91.9 \%$ to $96.5 \%$ ). There were 32 positives and 367 negatives by TPPA confirmatory testing. Overall sensitivity compared to TPPA was $43.8 \%$ (95\% CI $27.5 \%$ to $61.1 \%$ ) and specificity was $94.8 \%$ (95\% CI $92.2 \%$ to $96.8 \%)$.

Conclusions The Syph-Check POC test was moderately accurate compared to the RPR test, but not as sensitive compared to TPPA. A more accurate POC test for syphilis could be useful for clinicians to test clinic patients and provide immediate screening results for syphilis to patients during an office or clinic visit.

\section{P3-S6.05 COMPARING THE ANALYTICAL SENSITIVITIES OF SIX TREPONEMAL TESTS}

doi:10.1136/sextrans-2011-050108.479

H Jost, S Kikkert, Y Fakile, A Castro, T Ye, A Zaidi, R Ballard, D Cox. Centers for Disease Control and Prevention, Atlanta, USA

Background Traditional syphilis testing consists of screening with a non-treponemal test (RPR) and confirmation with a treponemal test (TP-PA, FTA-ABS, EIA, CIA, etc). Recently, that testing algorithm has been reversed due to efforts to reduce labour costs and the availability of automatable tests (EIA, CIA). Large numbers of discordant test results (treponemal + , non-treponemal-) can be obtained using the reverse algorithm and can be due to (1) treated cases of syphilis, (2) a false-positive treponemal test, or (3) a case of early primary syphilis that has yet to seroconvert. Those sera need to be confirmed with a second treponemal test to eliminate false positive specimens. The dilemma arises which treponemal test is best suited for confirmation, and secondly what are the relative analytical sensitivities of available treponemal tests used for both screening and confirmation.

Methods Two hundred randomised TP-PA positive, cleanascite treated samples (GADPH) were serially diluted with normal human sera to determine the analytical endpoint and sensitivity of six commonly used treponemal tests (TP-PA, FTA-ABS, TrepSure, TrepChek, TrepID, and LIAISON). All dilutions were treated as neat sera in each test, and the tests were performed according to the manufacturer's instructions. 